\title{
A Murine Model of the Eosinophilia-Myalgia Syndrome Induced by 1,1'-Ethylidenebis(L-Tryptophan)
}

\author{
Richard M. Silver, * Anna Ludwicka, * Marta Hampton, ${ }^{*}$ Takashi Ohba, " Sarah A. Bingel, ${ }^{3}$ Timothy Smith, \\ Russell A. Harley, ${ }^{*}$ John Maize, ${ }^{\star}$ and Melvyn P. Heyes" \\ From the Division of Rheumatology and Immunology, Departments of ${ }^{*}$ Medicine, ${ }^{\ddagger}$ Dermatology and Pathology, and ${ }^{\S}$ Laboratory \\ Animal Resources of the Medical University of South Carolina, Charleston, South Carolina 29425; and "Laboratory of Clinical Science, \\ Section on Analytical Biochemistry, National Institute of Mental Health, Bethesda, Maryland 20892
}

\begin{abstract}
The eosinophilia-myalgia syndrome (EMS) is a recently described disease that has been associated with the ingestion of L-tryptophan containing trace amounts of several impurities. The first such contaminant to be identified and linked epidemiologically to the EMS epidemic was 1,1'-ethylidenebis(L-tryptophan) (EBT), but its role in the etiology and pathogenesis of the syndrome has been controversial. We report the development of inflammation and fibrosis affecting the dermis and subcutis, including the fascia and perimyseal tissues, after the daily intraperitoneal administration of EBT to female C57BL/ 6 mice. Such changes are accompanied by increased numbers of mast cells, many of which appear to be degranulating. Plasma levels of quinolinic acid, a metabolic product of L-tryptophan via the kynurenine pathway, are reduced initially, and then become elevated when inflammation and fibrosis are more pronounced. The nature and location of the inflammatory cell infiltrate and fibrosis, as well as the presence of mast cells and alterations of L-tryptophan metabolism, are consistent with findings reported in patients with EMS. This murine model suggests that EBT may have been one of the mediators of EMS and should facilitate studies of the pathogenesis of EMS. ( $J$. Clin. Invest. 1994. 93:1473-1480.) Key words: EMS • L-tryptophan • EBT $\bullet$ fibrosis $\bullet$ mast cell
\end{abstract}

\section{Introduction}

The eosinophilia-myalgia syndrome (EMS) ${ }^{1}$ occurred as an epidemic in the United States in 1989 (1-3). During the epidemic the following surveillance case definition was established by the Centers for Disease Control (CDC): (a) blood eosinophil count $>10^{9} /$ liter; $(b)$ generalized myalgia of sufficient severity to limit a patient's usual activities; and $(c)$ exclusion of neoplasm or infection to account for symptoms (4).

This work was presented in part at the Annual and Southeast Regional Meetings of the American College of Rheumatology, October 12-15, 1992, Atlanta, GA, and March 26-28, 1993, Arlington, VA, respectively.

Address correspondence to Dr. Richard M. Silver, Professor of Medicine and Pediatrics, Medical University of South Carolina, Room 912, Clinical Sciences Building, 171 Ashley Avenue, Charleston, SC 29425.

Received for publication $10 \mathrm{July} 1993$ and in revised form 29 October 1993.

1. Abbreviations used in this paper: EBT, 1,1'-ethylidenebis(L-tryptophan); EMS, eosinophelia-myalgia syndrome; GVHD, graft-vs.-host disease; TOS, toxic oil syndrome.

The Journal of Clinical Investigation, Inc.

Volume 93, April 1994, 1473-1480
More than 1,500 cases and 38 deaths that fulfill the CDC criteria for EMS have been reported from the United States (5), but many more individuals who did not fulfill the CDC criteria or were not reported to the CDC are believed to have been affected (6).

In addition to eosinophilia and myalgia, many EMS patients developed scleroderma-like skin changes frequently accompanied by fasciitis $(2,3,7,8)$, peripheral neuropathy $(9$ 11 ), and pneumonitis (12). Remarkably similar clinical and histological changes were observed in patients affected by the toxic oil syndrome (TOS) that occurred in Spain in 1981 (13). In each syndrome, epidemiologic studies showed a strong association with the ingestion of products containing contaminants. Adulterated rapeseed oil was implicated in TOS (14), although a precise etiologic agent was never identified. The EMS epidemic has been linked to the ingestion of L-tryptophan manufactured by a single company $(15,16)$ and shown to contain several contaminants (16). The first to be identified was 1,1'-ethylidenebis(L-tryptophan) (EBT) (17). Recently, a second contaminant of L-tryptophan also linked to the EMS epidemic was identified as 3-(phenylamino)alanine (18), which resembles a putative contaminant of adulterated rapeseed oil implicated in TOS (19).

Although epidemiologic studies support a link between these contaminants and EMS, it is not known if they are the etiologic agent(s) or simply markers for a yet to be identified agent. Animal studies have been controversial (20), but one group has reported that female Lewis (LEW/N) rats fed Ltryptophan implicated in the EMS epidemic or LEW/N rats fed synthesized EBT develop myofascial fibrosis similar to that observed in patients with EMS $(21,22)$. In this study we succeeded in developing an animal model of EMS and report the induction of inflammation and fibrosis in skin and fascia of female C57BL/ 6 mice treated with daily intraperitoneal injections of EBT. In addition, we report the presence of increased numbers of mast cells similar to that observed in patients with EMS (23). Furthermore, since altered L-tryptophan metabolism and elevated plasma quinolinic acid levels were reported in patients with EMS (2), we investigated the effect of EBT administration on kynurenine pathway metabolism in this murine model.

\section{Methods}

Animals. 6-wk-old female C57BL/6 mice (The Jackson Laboratory, Bar Harbor, ME) weighing 20-22 g were maintained in animal quarters designed for pathogen-free mice and provided with food (Teklad Rodent Diet; Harlan Teklad Laboratory, Indianapolis, IN) and water ad libitum. All studies were conducted under a protocol approved by the institutional review board for animal studies.

Treatments. Groups of 10-12 animals received daily intraperitoneal injections consisting of $0.3 \mathrm{ml}$ of the following substances: (a) 

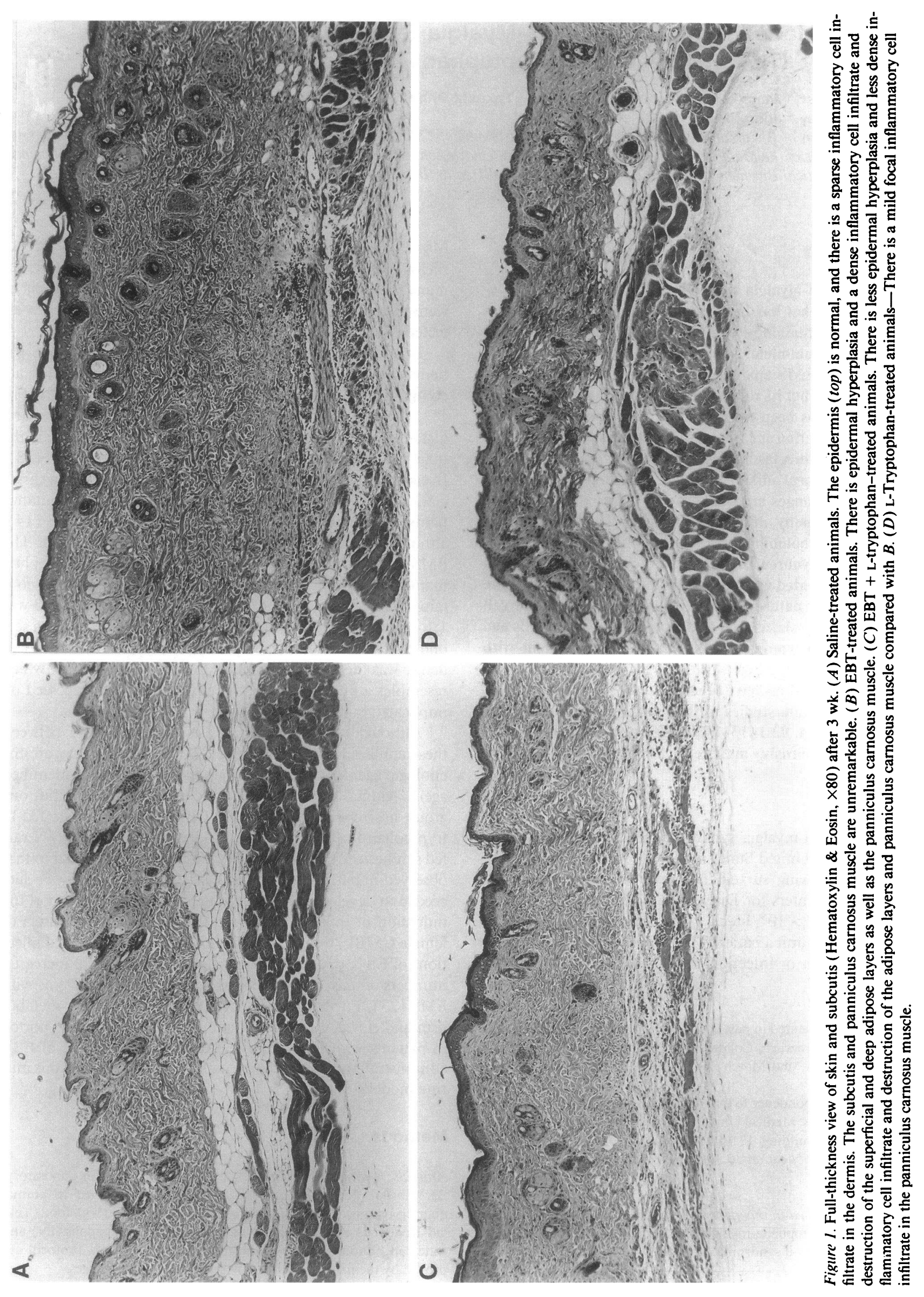
saline; (b) L-tryptophan (Sigma Chemical Co., St. Louis, MO), 30 $\mathrm{mg} / \mathrm{kg}$ body weight dissolved in saline; $(c)$ synthesized EBT (lot no. 901015 , kindly provided by Showa Denko K. K., Tokyo, shown by HPLC to have a purity of $98 \%$, as previously reported [24]) $40 \mu \mathrm{g} / \mathrm{kg}$ body weight dissolved in saline; and $(d)$ L-tryptophan combined with EBT (same doses as above). The doses of EBT and L-tryptophan were selected because they fall within the range of those ingested by patients with $\operatorname{EMS}(2,18,25)$. At various time intervals, ranging from $3 \mathrm{~d}$ to 6 wk, mid-morning blood and plasma were obtained and animals were killed by exsanguination under ketamine anesthesia. The following tissues were obtained for further analysis: $(a)$ skin and subcutaneous tissue from the interscapular area; $(b)$ muscle from the proximal hindlimb; $(c)$ lungs; $(d)$ heart; $(e)$ liver; and $(f)$ kidneys.

Pathology. Peripheral blood eosinophil counts were performed on blood smears prepared with Wright's stain. Portions of all tissues were fixed in $10 \%$ buffered formalin, embedded in paraffin, and stained with hematoxylin-eosin and Masson's trichrome stains. Lungs from seven animals in each treatment group were inflated with $3 \mathrm{ml}$ of $10 \%$ buffered formalin, fixed in the same solution at $4^{\circ} \mathrm{C}$ overnight, embedded in paraffin, and stained as above. Skin and subcutaneous tissue were also prepared with a specific esterase mast cell stain using naphthol AS-D chloroacetate and hematoxylin counterstain (26). Muscle tissue taken from the proximal hind limb was quick-frozen in liquid nitrogen-cooled isopentane. Mounted sections were cut at $5 \mu \mathrm{m}$ using a rotary microtome/cryostat. Sections were picked up on cover slips and stained with hematoxylin-eosin. All slides were coded and provided to investigators who were masked to the experimental conditions.

Biochemistry. Plasma quinolinic acid levels were determined using gas chromatography/mass spectrometry as previously described (27). All samples were run as coded specimens within a single assay.

Data analysis. For analysis of skin and subcutaneous tissues, two dermatopathologists (M. Hampton and J. Maize) independently graded tissue sections according to extent and severity of inflammation, destruction of cells, and fibrosis. An index of inflammation or fibrosis was calculated on the basis of the severity ( none $=0$; mild $=1$; moderate $=2$; severe $=3$ ) $\times$ extent $($ focal $=1$; diffuse $=3$ ). The index of inflammation or fibrosis varied up to $20 \%$ between experiments, each consisting of 10-12 animals/treatment group, and data from individual experiments are presented in each figure and table unless indicated otherwise. Mast cells were counted under high magnification ( $\times 200)$ with a light microscope equipped with a micrometer and are expressed as the number of mast cells per $1.5 \mathrm{~mm}^{2}$. A neuropathologist (T. Smith) graded muscle sections for thickness of fascia $(\mu \mathrm{m})$, necrotic and atrophic myocytes (no./2.5 $\mathrm{mm}^{2}$ ), and presence or absence of islands of perimyseal inflammatory cells, perineural inflammation, and fibrosis. Group means \pm standard deviation were calculated for his-

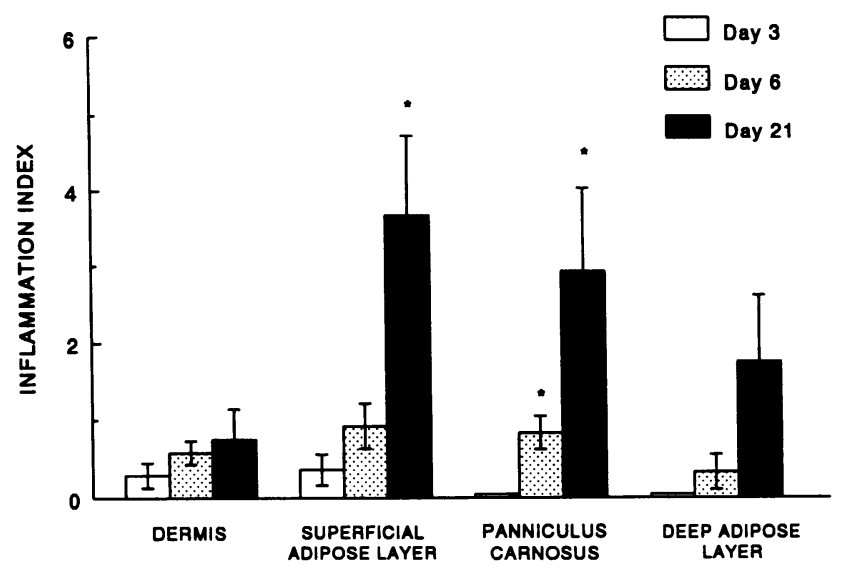

Figure 3. Time-related effect of EBT on inflammation. Focal and mild inflammation at day 3 evolve to more diffuse and severe inflammation by $21 \mathrm{~d}$, particularly in the superficial adipose layer and panniculus carnosus muscle $\left({ }^{*} P<0.01\right)$.

topathologic parameters and quinolinic acid. For comparisons among animals treated with saline, L-tryptophan, or EBT, Kruskal-Wallis nonparametric ANOVA test with Dunn's multiple correction test was used.

\section{Results}

Clinical observations. In the course of several sets of experiments involving $\geq 40$ animals each, no consistent effect on weight, coat appearance, or gross motor behavior was noted for any of the treatment modalities. No significant mortality occurred over the course of $6 \mathrm{wk}$ of treatment. Blood smears obtained at intervals ranging from $3 \mathrm{~d}$ to $6 \mathrm{wk}$ of treatment showed no significant eosinophilia in any of the treatment groups (data not shown).

Skin and subcutaneous histopathology. The administration of EBT was accompanied by a mononuclear cell infiltrate in the dermis and subcutis of most animals, with hyperplasia of the epidermis seen in as many as $20 \%$ of the animals (Fig. 1). Moderate to severe inflammation was present throughout the dermis and all layers of the subcutis of animals exposed to

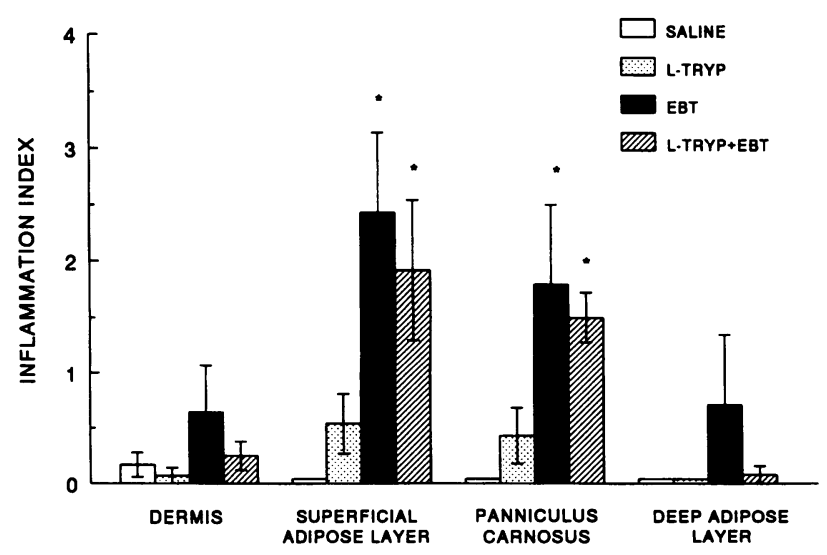

Figure 2. Inflammation index. Significantly greater inflammation is present in animals treated for 3 wk with EBT or EBT + L-tryptophan than in control animals treated with saline or L-tryptophan $(L$ $T R Y P)$ alone $\left({ }^{*} P<0.01\right)$.

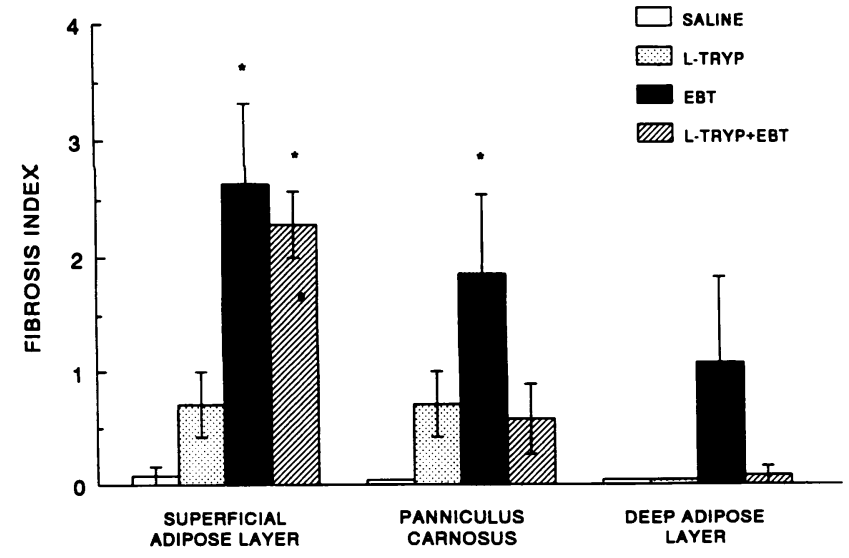

Figure 4. Fibrosis index. Significantly greater fibrosis is present in animals treated for 3 wk with EBT or EBT + L-tryptophan compared with control animals treated with saline or L-tryptophan $(L-T R Y P)$ alone $\left({ }^{*} P<0.01\right)$. 


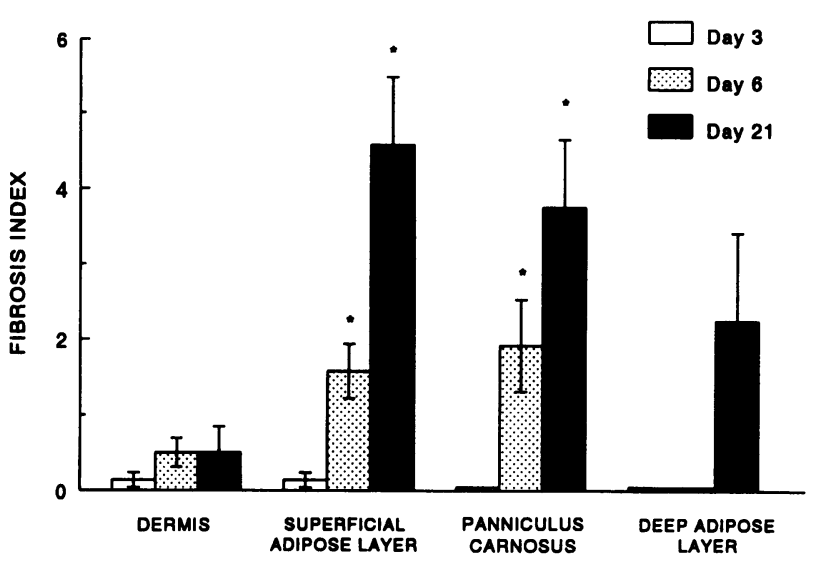

Figure 5. Time-related effect of EBT on fibrosis. Significant fibrosis is evident in the superficial adipose layer and panniculus carnosus muscle by day 6 , increasing by day 21 ( $^{*} P<0.01$ compared with day 3 ).

EBT, with the most extensive inflammation occurring in the superficial adipose layer and the panniculus carnosus muscle (Figs. 1 and 2). The cellular infiltrate was composed predominantly of lymphocytes and monocytes with occasional plasma cells and frequent mast cells (see below). Focal sites of inflammation were observed in the dermis and subcutis of animals treated with saline or L-tryptophan (Fig. 1), but such changes were never as severe as those observed in animals exposed to EBT. Focal cellular infiltrates were observed as early as $3 \mathrm{~d}$ after beginning EBT administration; diffuse changes were present in the subcutis after $6 \mathrm{~d}$ of treatment and were more prevalent and severe after 3 wk (Fig. 3). Of interest was the observation that animals treated with L-tryptophan + EBT had less inflammation than did animals treated with EBT alone (Figs. 1 and 2).

Cellular infiltration was accompanied by varying degrees of fibrosis and destruction of the adipose layers and panniculus carnosus muscle. As was the case with inflammation, such changes were always more prevalent and severe in animals exposed to EBT than in animals exposed to saline, L-tryptophan, or EBT combined with L-tryptophan (Figs. 4 and 5). With EBT administration fibrotic changes were observed in all layers of the subcutis, and some animals developed diffuse fibrosis in the dermis also. Fibrosis was less frequent and severe in animals treated with L-tryptophan + EBT than in animals treated with EBT alone (Fig. 4). The fibrosis of the dermis and subcu- tis evolved from focal to diffuse by 3 wk of exposure to EBT (Fig. 5).

EBT exposure was accompanied by a two- to nearly fourfold increase in the number of dermal and subcutaneous mast cells (Table I). The increase in mast cell number was evident after $3 \mathrm{~d}$ of exposure to EBT, when significant increases were noted in the dermis, superficial adipose layer, and the panniculus carnosus muscle (Table I and Fig. 6). In the control group of saline-treated animals, mast cells were limited primarily to the dermis. After $6 \mathrm{~d}$ of EBT exposure the mast cell numbers in some skin layers appeared to decline to the control level observed in saline-treated animals and were significantly lower than at day 3. By $6 \mathrm{wk}$, the number of mast cells increased to levels comparable to or greater than day 3 .

Muscle and fascia. The endomysium, perimysium, and fascia of animals exposed to EBT showed progressive thickening with increasing treatment times (Table II). After 6 wk of exposure to EBT there was a significant increase in the thickness of the fascia of the muscle as compared with animals treated with saline $(168 \pm 22 \mu \mathrm{m}$ vs. $43 \pm 5 \mu \mathrm{m} ; P<0.01)$ or L-tryptophan $(168 \pm 22 \mu \mathrm{m}$ vs. $69 \pm 7 \mu \mathrm{m} ; P<0.01)$ (Table II). L-Tryptophan alone resulted in a slight increase in fascia thickness compared with animals receiving saline, but the difference was not statistically significant.

Muscle from all treatment groups showed nonspecific myopathic changes consisting of basophilic myocytes, atrophic myocytes, and myocytes with central nuclei. A significant increase in the number of necrotic muscle fibers was seen only in the group of animals receiving EBT for $4 \mathrm{wk}(2.92 \pm 0.95 / 2.5$ $\left.\mathrm{mm}^{2}\right)$ compared with animals receiving saline $(0.18 \pm 0.13 / 2.5$ $\left.\mathrm{mm}^{2}\right)$ or L-tryptophan $\left(0.27 \pm 0.19 / 2.5 \mathrm{~mm}^{2}\right)(P<0.01$ each $)$, but not at 6 wk. After 4 wk of EBT exposure there was also an increase in the number of atrophic myocytes compared with controls, but this was not apparent at $6 \mathrm{wk}$. Inflammatory islands were rarely identified within muscle tissue. Muscle spindles were only rarely inflamed. Endothelial cell hyperplasia was present in the endomysial septa of some but not all animals treated with EBT for 6 wk.

Other pathology. No significant abnormalities of the heart, kidney, or liver were detected by light microscopy. Focal areas of inflammation and fibrosis were observed in the lungs of animals treated for $3 \mathrm{wk}$ with L-tryptophan alone or in combination with EBT, but such changes were mild and there was no significant difference in the inflammation index or fibrosis index among the various control and treatment groups. Areas of

Table I. Mast Cell Numbers

\begin{tabular}{llcccc}
\hline \multicolumn{1}{c}{ Layer } & Exp. condition & Day 3 & Day 6 & Day 21 & Day 42 \\
\hline Dermis & Saline & $79.3 \pm 5.1^{*}$ & $130.4 \pm 12.8$ & $79.7 \pm 6.1$ & ND $^{\ddagger}$ \\
& EBT & $157.9 \pm 14.8^{3}$ & $120.2 \pm 16.9^{\| 1}$ & $98.3 \pm 15.6$ & $162.7 \pm 9.6$ \\
Superficial Adipose & Saline & $29.4 \pm 6.2$ & $60.4 \pm 3.1$ & $50.2 \pm 5.7$ & ND \\
& EBT & $99.5 \pm 12.4^{\S}$ & $56.5 \pm 14.3^{\| 1}$ & $61.3 \pm 7.3$ & $77.9 \pm 4.7$ \\
Panniculus Carnosus & Saline & $1.4 \pm 0.5$ & $12.0 \pm 3.7$ & $11.5 \pm 2.6$ & ND \\
& EBT & $5.2 \pm 1.5 \pi$ & $10.2 \pm 1.8$ & $12.0 \pm 2.7$ & $16.5 \pm 3.5^{* *}$ \\
Deep Adipose & Saline & $2.2 \pm 1.0$ & $9.4 \pm 3.4$ & $4.5 \pm 1.1$ & ND \\
& EBT & $4.2 \pm 0.8$ & $7.2 \pm 1.4$ & $7.0 \pm 1.4$ & $17.0 \pm 2.4^{* *}$ \\
\hline
\end{tabular}

${ }^{*}$ Number of mast cells per linear $1.5 \mathrm{~mm}^{2}$ of each layer, mean \pm SD. ${ }^{\ddagger} \mathrm{ND}$, not done. ${ }^{\S} P<0.01 \mathrm{EBT}$ vs. saline, day 3 . " $P<0.05$ day 3 vs. day 6, EBT. ' $P=0.05$ EBT vs. saline, day 3 . ${ }^{* *} P<0.01$ day 3 vs. day 42 , EBT. 


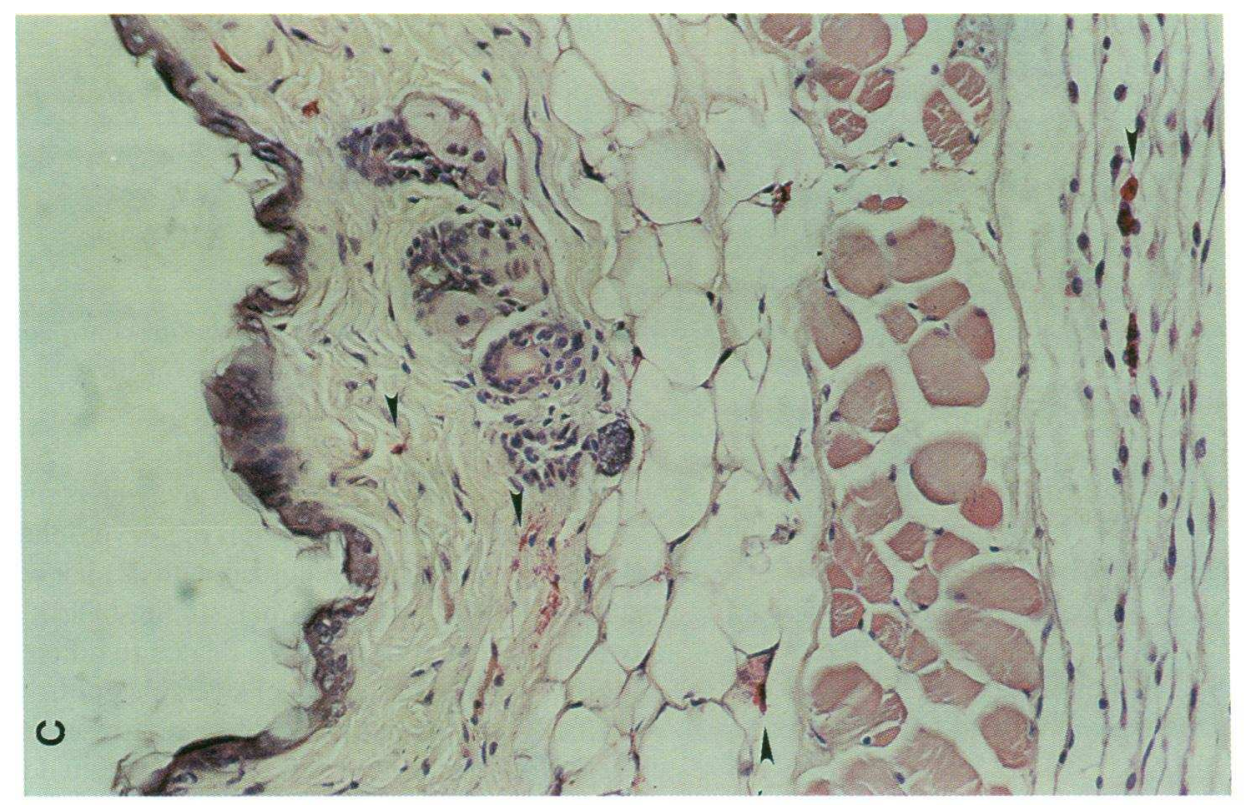

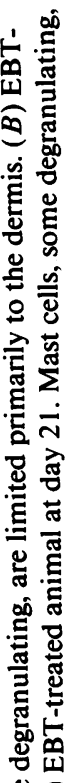

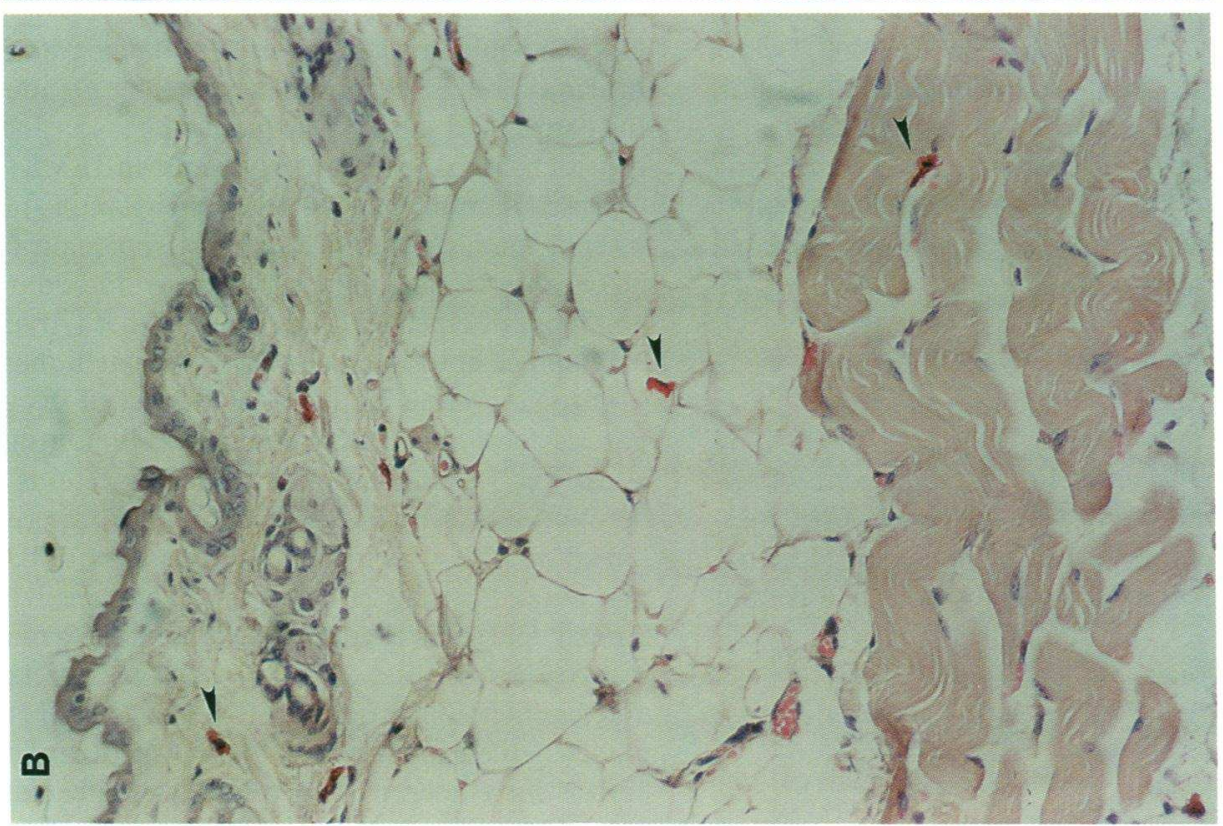

言

这

过

苋产

卷

吾骂

उ.

홍

悹

ॠึ

宅:

的

巡

动

丞
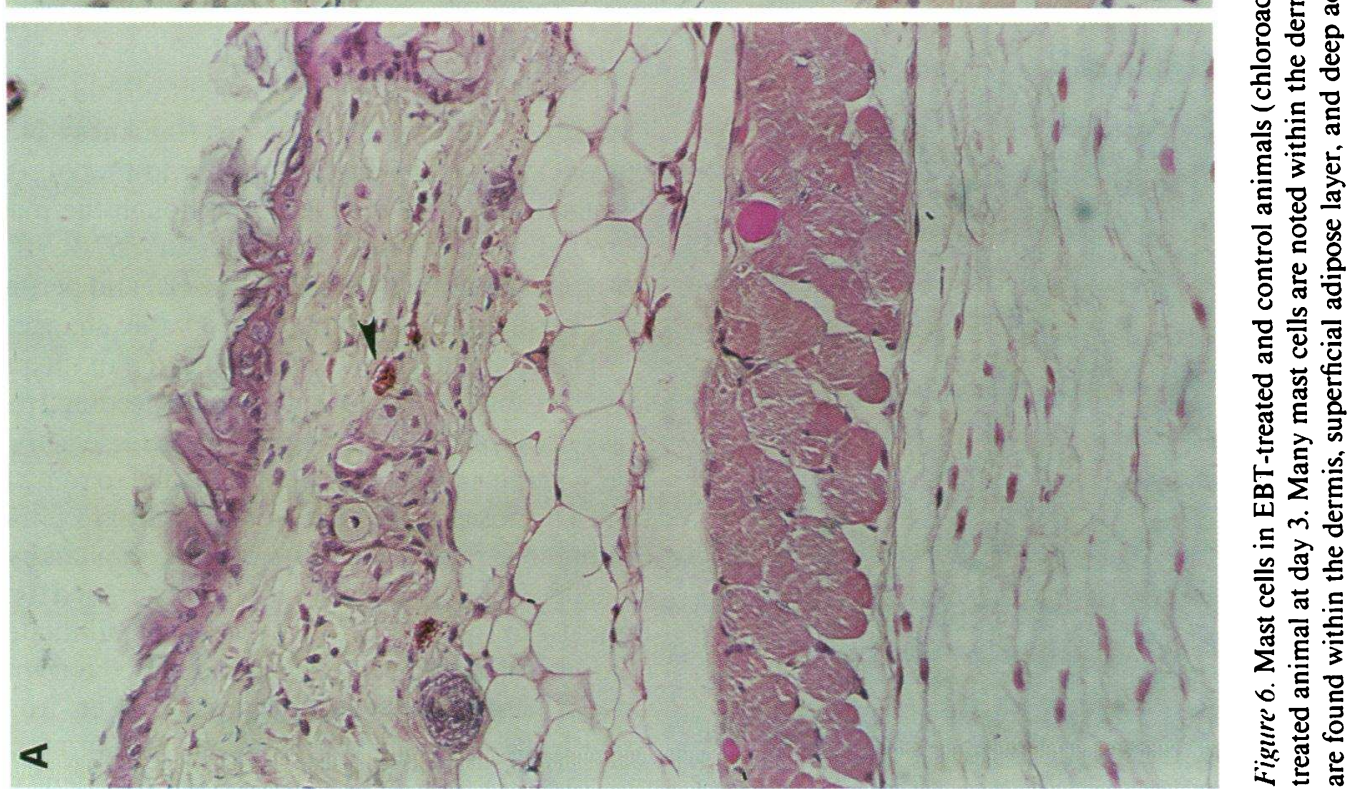
Table II. Fascia and Muscle Histopathology of EBT-treated and Control Mice

\begin{tabular}{lcccrr}
\hline & Saline* & L-Tryp* & EBT (3 wk) & EBT (4 wk) & EBT (6 wk) \\
\hline Fascia thickness $(\mu \mathrm{m})$ & $43 \pm 5$ & $69 \pm 7$ & $47 \pm 7$ & $72 \pm 6$ & $168 \pm 22^{8 ॥}$ \\
Atrophic muscle fibers $\left(\right.$ no. $\left./ 2.5 \mathrm{~mm}^{2}\right)$ & $2.07 \pm 0.86$ & $2.00 \pm 0.56$ & $2.25 \pm 0.31$ & $3.67 \pm 0.94$ & $3.07 \pm 0.61$ \\
Necrotic muscle fibers $\left(\right.$ no. $\left./ 2.5 \mathrm{~mm}^{2}\right)$ & $0.18 \pm 0.13$ & $0.27 \pm 0.19$ & $0.50 \pm 0.34$ & $2.92 \pm 0.95^{8 \|}$ & $0.15 \pm 0.10$
\end{tabular}

${ }^{*}$ Data pooled from 3- and 6-wk experiments. ${ }^{\ddagger}$ L-Tryp, L-tryptophan. Significantly different $(P<0.01)$ from ${ }^{8}$ saline control or "L-tryptophan control.

lymphoid tissue within the lungs were seen in animals receiving EBT or the combination of EBT + L-tryptophan, but such changes were always focal and mild.

Kynurenine pathway metabolites. Plasma quinolinic acid levels remained stable in saline-treated animals. There was a biphasic response of plasma quinolinic acid after administration of EBT (Fig. 7). Plasma quinolinic acid fell from a baseline value of $206 \pm 62$ to $110 \pm 24$ and $131 \pm 35 \mathrm{nM}$ after 3 and $6 \mathrm{~d}$ of EBT, respectively $(P<0.001$ compared with saline controls). Quinolinic acid returned toward baseline by $2 \mathrm{wk}$ of EBT exposure $(180 \pm 55 \mathrm{nM} ; P=\mathrm{NS}$ compared with saline controls) and continued to rise to levels that were significantly elevated compared with baseline or saline-treated control animals by 3 wk $(326 \pm 71 \mathrm{nM} ; P<0.001)$. The levels began to return toward baseline by 4 and 6 wk of EBT exposure but remained high $(299 \pm 96$ and $253 \pm 45 \mathrm{nM}$, respectively; $P$ $<0.05$ each)

\section{Discussion}

The ingestion of L-tryptophan containing trace amounts of several contaminants has been shown by a number of epidemiologic studies to be associated with the EMS epidemic $(15,16)$. An etiologic role for EBT, the contaminant initially linked to

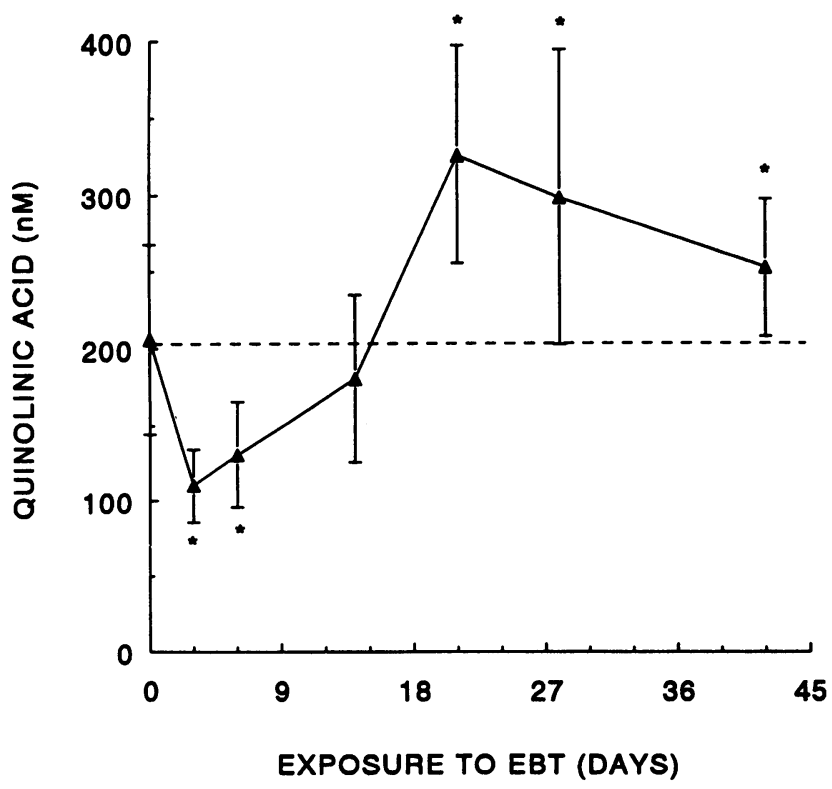

Figure 7. Effect of EBT on quinolinic acid. Plasma concentration (nM) of the kynurenine pathway metabolite quinolinic acid in mice treated with EBT for $3 \mathrm{~d}$ to $6 \mathrm{wk} .{ }^{*} P<0.05$ compared with salinetreated animals (-.-.--). the epidemic, has been suggested by studies of female Lewis (LEW/N) rats fed implicated lots of L-tryptophan or synthetic EBT, in which myofascial fibrosis with (21) or without inflammation (22) was observed. Such studies have not been generally reproducible (20), perhaps related to differences in methodology, so there has been considerable controversy over the role of EBT in the etiology and pathogenesis of EMS. In the current study, we demonstrate that significant dermal and subcutaneous inflammation and fibrosis occur when female C57BL/ 6 mice are administered synthetic EBT by intraperitoneal injection. EBT appears to be capable of inducing lesions of the dermis and subcutis, including the fascia and perimysium, consistent with those seen in patients with $\operatorname{EMS}(2,3,7,8,11)$. Other contaminants, such as 3-(phenylamino)alanine, linked by epidemiologic studies to the EMS epidemic (18), may also be capable of inducing such changes. In addition, our study and those of others $(21,22)$ indicate that L-tryptophan per se may induce fibrosis, albeit to a lesser extent than EBT.

In this murine model of EBT-induced inflammation and fibrosis, focal lesions were observed in the dermis and subcutis as early as $3 \mathrm{~d}$ after initiation of EBT injections and tended to become diffuse by 3-6 wk. Epidermal hyperplasia was observed in $20 \%$ of animals as early as day 6 , becoming more pronounced by day 21 (see Fig. 1), and resolving after $6 \mathrm{wk}$. Similar epidermal changes were reported in a murine model of graft-vs.-host disease (GVHD), where regression was also noted as the cellular infiltrate lessened (28). It is possible than in each of these murine models of cutaneous inflammation and fibrosis the epidermal hyperplasia and subcutaneous fibrosis are mediated by growth factors and cytokines secreted by inflammatory cells.

Fascial thickness was increased fourfold after 6 wk of EBT exposure. The degree of fascial thickness observed in the current study is remarkably similar to that observed in LEW/ $\mathrm{N}$ rats by Crofford et al. (21), although the magnitude of inflammation appears to be greater in C57BL/6 mice. As was observed in patients with EMS, fascial and perimyseal changes were more pronounced than myopathic changes per se.

The daily dose of EBT administered in the present study is less than that of previously reported feeding studies (20-22), $40 \mu \mathrm{g} / \mathrm{kg}$ vs. $40 \mathrm{mg} / \mathrm{kg}$, suggesting species sensitivity or, alternatively, greater systemic exposure related to route of administration. The doses used in the present study are within the range of estimated exposure to EBT and L-tryptophan by patients who developed EMS $(2,18,25)$. It might be argued that the intraperitoneal administration of EBT does not simulate the exposure of individuals who ingested contaminated L-tryptophan per os, since it has been shown that in an acid environment similar to gastric fluid EBT breaks down to L-tryptophan and two intermediate products, 1-(hydroxyethylidene)-L-tryptophan 
(HET) and 1-methyl-1,2,3,4-tetrahydro- $\beta$-carboline-3-carboxylic acid (MCAA) $(24,29)$. Nevertheless, the breakdown of EBT in an acid milieu is not too fast to preclude some absorption of EBT into the circulation (29). Furthermore, concomitant ingestion of supraphysiologic quantities of L-tryptophan might have altered the intestinal epithelial border (30), perhaps facilitating absorption of EBT.

Eosinophilia was not observed in the current study or in those involving the LEW/N rat (20-22). Eosinophils were reported to be increased in the blood of BALB/c mice administered EBT-contaminated-L-tryptophan by intraperitoneal injection in an oil vehicle, but control animals receiving unimplicated L-tryptophan also developed eosinophilia (31). Although eosinophils and eosinophil-derived proteins have been postulated to play a role in the pathophysiology of EMS $(1)$, this and other studies $(21,22)$ suggest that significant inflammation and fibrosis may occur after EBT exposure without an increase in blood or tissue eosinophils.

In the present murine model, dermal and subcutaneous mast cells were noted to be increased as early as $3 \mathrm{~d}$ after EBT exposure. Oral feeding of L-tryptophan containing EBT to LEW/ $\mathrm{N}$ rats also was associated with an increase in the number of degranulating mast cells within the intestinal mucosa (32) and in the dermis, fascia, and muscle (L. Love, personal communication). The apparent decline in mast cell number observed after $6 \mathrm{~d}$ in the current study may represent degranulation of mast cells, since marked extracellular staining was apparent as early as day 3 . By $6 \mathrm{wk}$, dermal and superficial subcutaneous mast cell numbers were comparable to mast cell levels at day 3 , whereas deeper subcutaneous mast cell numbers remained significantly elevated. In a murine model of chronic GVHD, Claman et al. (33) showed that dermal mast cells seemingly disappear due to degranulation and loss of intracellular granules, yet "phantom mast cells" can be identified by ultrastructural methods. The changes observed in the present model are consistent with those observed in skin sections above the level of the panniculus carnosus in the murine model of GVHD (34). Mast cells are prominent in many chronic fibrosing disorders, including scleroderma $(35,36)$. Furthermore, mast cells were noted to be increased in the skin of patients with EMS (37) and a similar condition, TOS (36). The role of the mast cell in the pathogenesis of fibrosis remains unclear, but the presence of degranulating mast cells in fibrotic lesions of mice treated with EBT is additional evidence that the current model resembles EMS.

Of interest is our observation that inflammation and fibrosis were less intense when animals were treated with EBT plus L-tryptophan compared with EBT alone. One explanation for this observation is that L-tryptophan and EBT compete for binding at unknown but important sites. Although it has been reported that EBT does not inhibit the binding of L-tryptophan to hepatic nuclear envelopes (38), EBT and L-tryptophan may compete for incorporation into proteins. The latter effect has been shown to be due to direct competition rather than an inhibitory effect of EBT on in vitro protein synthesis $(\mathrm{H}$. Sidransky, personal communication). It is also possible that Ltryptophan may modulate the effect of EBT on connective tissue metabolism. TGF- $\beta_{1}$ has been shown to be present in EMS lesions and is postulated to play a role in the development of the connective tissue alterations present in EMS-associated fasciitis (39). In vitro studies of human dermal fibroblasts suggest that L-tryptophan can block the stimulatory effect of TGF- $\beta$ on collagen synthesis (V. Falanga, personal communication) and can upregulate collagenase gene expression (40). Animal studies reported here and by others $(21,22)$, however, suggest that L-tryptophan per se induces rather than inhibits fibrosis in vivo. The serotonin pathway of L-tryptophan metabolism may be an important mediator of such fibrosis, since serotonin has been shown to induce fibrosis in vivo (41) and fibroblast proliferation in vitro $(42,43)$.

Plasma quinolinic acid levels were significantly reduced in the first week of EBT exposure. Such a decrease in kynurenine pathway metabolism might be explained by loss of tryptophan dioxygenase activity due to a hepatotoxic effect of EBT or one of its breakdown products, yet no significant hepatic abnormalities were observed at the light microscopic level. Alternatively, competitive inhibition of tryptophan dioxygenase by EBT might explain the observed decline in quinolinic acid levels in the early days after EBT exposure. The significant elevation in quinolinic acid observed later, when inflammation and fibrosis were more pronounced, is similar to what we reported in patients during the active phase of EMS $(2,23)$, and may be the result of induction of indoleamine 2,3-dioxygenase by interferons (44). The present finding of decreased plasma quinolinic acid at the earliest time points followed subsequently by elevated quinolinic acid may indicate that quinolinic acid is not of primary importance in the pathogenesis of EMS, but merely a marker of ongoing inflammation.

The availability of a model for EMS using an animal strain without inherent immunologic abnormalities should facilitate studies of the early cellular events central to the pathogenesis of EMS. Such studies may lead to a better understanding of EMS and perhaps related conditions, such as eosinophilic fasciitis and TOS. Ultimately such studies may lead to the development of more effective treatment strategies for such diseases.

\section{Acknowledgments}

We acknowledge the expert assistance of P. Briggs, K. Prioleau, T. Ruth, J. Nicholson, and A. Donaldson.

This work was supported by grants from K. K. Showa Denko (Tokyo, Japan ) and the Medical University of South Carolina Institutional Research Funds (Charleston, SC).

\section{References}

1. Hertzman, P. A., W. L. Blevins, J. Mayer, B. Greenfield, M. Ting, and G. J. Gleich. 1990. Association of the eosinophilia-myalgia syndrome with the ingestion of tryptophan. N. Engl. J. Med. 322:869-873.

2. Silver, R. M., M. P. Heyes, J. C. Maize, B. Quearry, M. Vionnet-Fuasset, and E. M. Sternberg. 1990. Scleroderma, fasciitis, and eosinophilia associated with the ingestion of L-tryptophan. $N$. Engl. J. Med. 322:874-881.

3. Clauw, D. J., D. J. Nashel, and P. Katz. 1990. Tryptophan-associated eosinophilic connective-tissue disease. A new clinical entity? J. Am. Med. Assoc. 263:1502-1506.

4. Kilbourne, E. M., L. A. Swygert, R. M. Philen, R. K. Sun, S. B. Auerbach, L. Miller, D. E. Nelson, and H. Falk. 1990. Interim guidance on the eosinophiliamyalgia syndrome. Ann. Int. Med. 112:85-87.

5. Auerbach, S. B., and H. Falk. 1991. Eosinophilia-myalgia syndrome: CDC update. Clevel. Clin. J. Med. 58:215-217.

6. Ahmed, S. R., and D. Clauw. 1991. USA: EMS and L-tryptophan. Lancet. 338:1512.

7. Bulpitt, K. J., M. A. Verity, P. J. Clements, and H. E. Paulus. 1990. Association of L-tryptophan and an illness resembling eosinophilic fasciitis. Clinical and histopathologic findings in four patients with eosinophilia-myalgia syndrome. Arthritis Rheum. 33:918-929.

8. Varga, J., J. Peltonen, J. Uitto, and S. Jimenez. 1990. Development of diffuse fasciitis with eosinophilia during L-tryptophan treatment: demonstration 
of elevated type I collagen gene expression in affected tissues. A clinicopathologic study of four patients. Ann. Int. Med. 112:344-351.

9. Smith, B. E., and P. J. Dyck. 1990. Peripheral neuropathy in the eosinophilia-myalgia syndrome associated with L-tryptophan ingestion. Neurology. 40:1035-1040.

10. Heiman-Patterson, T., S. J. Bird, G. J. Parry, J. Varga, M. E. Shy, N. W. Culligan, L. Edelsohn, G. T. Tatarian, M. P. Heyes, C. A. Garcia, and A. J. Tahmoush. 1990. Peripheral neuropathy associated with eosinophilia-myalgia syndrome. Ann. Neurol. 28:522-528.

11. Kaufman, L. D., R. J. Seidman, and B. L. Gruber. 1990. L-Tryptophanassociated eosinophilic perimyositis, neuritis, and fasciitis. A clinicopathologic and laboratory study of 25 patients. Medicine (Baltimore). 69:187-199.

12. Read, C. A., D. Clauw, C. Weir, A. T. Da Silva, and P. Katz. 1992. Dyspnea and pulmonary function in the L-tryptophan-associated eosinophiliamyalgia syndrome. Chest. 101:1282-1286.

13. Kilbourne, E. M., M. Posada de la Paz, I. Abaitua Borda, M. D. Ruiz-Navarro, R. M. Philen, and H. Falk. 1991. Toxic oil syndrome: a current clinical and epidemiologic summary, including comparisons with eosinophilia-myalgia syndrome. J. Am. Coll. Cardiol. 18:711-717.

14. Kilbourne, E. M., M. Posada de la Paz, and I. Abaitua Borda. 1992. Epidemiological studies. In Toxic Oil Syndrome: Current Knowledge and Future Perspectives. WHO Regional Publications, European Series, Copenhagen. 42:525.

15. Slutsker, L., F. C. Hoesly, L. Miller, L. P. Williams, J. C. Watson, and D. W. Fleming. 1990. Eosinophilia-myalgia syndrome associated with exposure to tryptophan from a single manufacturer. J. Am. Med. Assoc. 264:213-217.

16. Belongia, E. A., C. W. Hedberg, G. J. Gleich, K. E. White, A. N. Mayeno, D. A. Loegering, S. L. Dunnette, P. L. Pirie, K. L. MacDonald, and M. T. Osterholm. 1990. An investigation of the cause of the eosinophilia-myalgia syndrome associated with tryptophan use. N. Engl. J. Med. 323:357-365.

17. Mayeno, A. N., F. Lin, C. S. Foote, D. A. Loegering, M. M. Ames, C. W. Hedberg, and G. J. Gleich. 1990. Characterization of "Peak E," a novel amino acid associated with eosinophilia-myalgia syndrome. Science (Wash. DC). 250:1707-1708.

18. Mayeno, A. N., E. A. Belongia, F. Lin, S. K. Lundy, and G. J. Gleich. 1992. 3-(Phenylamino)alanine, a novel aniline-derived amino acid associated with the eosinophilia-myalgia syndrome: a link to the toxic oil syndrome? Mayo Clin. Proc. 67:1134-1139.

19. Vazques Roncero, A., C. Janer del Valle, R. Maestro Duran, and E. Graciani Constante. 1983. New aniline derivatives in cooking oils associated with the toxic oil syndrome (letter to the editor). Lancet. ii:1024-1025.

20. Clauw, D. J., L. Scott, H. J. Manz, E. Kagan, and P. Katz. 1992. A rat feeding study using tryptophan implicated as causal in the eosinophilia myalgia syndrome (EMS). Arthritis Rheum. 35:25S. (Abstr.)

21. Crofford, L. J., J. I. Rader, M. C. Dalakas, R. H. Hill, Jr., S. W. Page, L. L. Needham, L. S. Brady, M. P. Heyes, R. L. Wilder, P. W. Gold, et al. 1990. L-Tryptophan implicated in human eosinophilia-myalgia syndrome causes fasciitis and perimyositis in the Lewis rat. J. Clin. Invest. 86:1757-1763.

22. Love, L. A., J. I. Rader, L. J. Crofford, R. B. Raybourne, M. A. Principato, S. W. Page, M. W. Trucksess, M. J. Smith, E. M. Dugan, M. L. Turner, et al. 1993. Pathological and immunological effects of ingesting L-tryptophan and 1,1'ethylidenebis(L-tryptophan) in Lewis rats. J. Clin. Invest. 91:804-811.

23. Silver, R. M., K. McKinley, E. A. Smith, B. Quearry, Y. Harati, E. M. Sternberg, and M. P. Heyes. 1992. Tryptophan metabolism via the kynurenine pathway in patients with the eosinophilia-myalgia syndrome. Arthritis Rheum. 35:1097-1105.

24. Ito, J., Y. Hosaki, Y. Torigoe, and K. Sakimoto. 1992. Identification of substances formed by decomposition of peak E substance in tryptophan. Food. Chem. Toxicol. 30:71-81.

25. Kamb, M. L., J. J. Murphy, J. L. Jones, J. C. Caston, K. Nederlof, L. F. Horney, L. A. Swygert, H. Falk, and E. M. Kilbourne. 1992. Eosinophilia-myalgia syndrome in L-tryptophan-exposed patients. J. Am. Med. Assoc. 267:77-82.

26. Leder, L. D. 1964. Uber die fermentcytochemische Darstellung von neu- trophilen myeloischen Zellen und Gewebsmastzellen im Paraffinschnitt. Klin. Wochenschr. 42:553.

27. Heyes, M. P., and S. P. Markey. 1988. Quantification of quinolinic acid in rat brain, whole blood, and plasma by gas chromatography and negative chemical ionization mass spectrometry: effects of systemic L-tryptophan administration on brain and CSF quinolinic acid. Anal. Biochem. 174:349-359.

28. Claman, H. N., B. D. Jaffee, J. C. Huff, and R. A. F. Clark. 1985. Chronic graft-versus-host disease as a model for scleroderma. II. Mast cell depletion with deposition of immunoglobulins in the skin and fibrosis. Cell. Immunol. 94:7384.

29. Driskell, W. J., D. L. Ashley, J. Grainger, S. R. Sirimanne, E. P. Mazzola, S. W. Page, L. L. Needham, and R. H. Hill, Jr. 1992. Identification of decomposition products of $1,1^{\prime}$-ethylidenebis [L-tryptophan], a compound associated with the eosinophilia-myalgia syndrome. Bull. Environ. Contam. Toxicol. 48:679687.

30. Madara, J., and S. Carlson. 1991. Supraphysiologic L-tryptophan elicits cytoskeletal and macromolecular permeability alterations in hamster small intestinal epithelium in vitro. J. Clin. Invest. 87:454-462.

31. Jones, M. M., X-D. Wu, R. Schelper, A. Bergold, and T. J. Waldschmidt. 1991. L-tryptophan induces pneumonitis and eosinophilia in BALB/c mice. Arthritis Rheum. 34:S116. (Abstr.)

32. DeSchryver-Kecskemeti, K., T. L. Gramlick, L. J. Crofford, J. I. Rader, S. W. Page, L. L. Needham, R. H. Hill, Jr., and E. M. Sternberg. 1991. Mast cell and eosinophil infiltration in intestinal mucosa of Lewis rats treated with L-tryptophan implicated in human eosinophilia myalgia syndrome. Mod. Pathol. 4:354-357.

33. Claman, H. N., K. L. Choi, W. Sujansky, and A. E. Vatter. 1986. Mast cell "disappearance" in chronic murine graft-vs-host disease (GVHD): ultrastructural demonstration of "phantom mast cells." J. Immunol. 137:2009-2013.

34. Claman, H. N. 1985. Mast cell depletion in murine chronic graft-versushost disease. J. Invest. Dermatol. 84:246-248.

35. Hawkins, R. A., H. N. Claman, R. A. F. Clark, and J. C. Steigerwald. 1985. Increased dermal mast cell populations in progressive systemic sclerosis: a link in chronic fibrosis? Ann. Int. Med. 102:182-186.

36. Fonseca, E., and J. Solis. 1985. Mast cells in the skin: progressive systemic sclerosis and the toxic oil syndrome. Ann. Int. Med. 102:864-865.

37. Kaufman, L., R. Seidman, M. Phillips, and B. Gruber. 1990. Cutaneous manifestations of the L-tryptophan-associated eosinophilia-myalgia syndrome: a spectrum of sclerodermatous skin disease. J. Am. Acad. Dermatol. 23:10631069.

38. Sidransky, H., E. Verney, J. W. Cosgrove, and A. M. Schwartz. 1992. Studies with compounds that compete with tryptophan binding to rat hepatic nuclei. J. Nutr. 122:1085-1095.

39. Peltonen, J., J. Varga, S. Sollberg, J. Uitto, and S. A. Jimenez. 1991. Elevated expression of the genes for transforming growth factor $\beta_{1}$ and type VI collagen in diffuse fasciitis associated with the eosinophilia-myalgia syndrome. $J$. Invest. Dermatol. 96:20-25.

40. Varga, J., L. Li, J. Jeffrey, H. Welgus, and S. Jimenez. 1992. Stimulation of metalloproteinase gene expression in vitro by L-tryptophan (LTRP). Arthritis Rheum. 35:S48. (Abstr.)

41. MacDonald, R. A., S. L. Robbins, and G. K. Mallory. 1958. Dermal fibrosis following subcutaneous injections of serotonin creatinine sulphate. Proc. Soc. Exp. Biol. Med. 97:334-337.

42. Boucek, R. J., and T. R. Alvarez. 1970. 5-Hydroxytryptamine: a cytospecific growth stimulator of cultured fibroblasts. Science (Wash. DC). 167:898899.

43. Seuwen, K., I. Magnaldo, and J. Pouyssegur. 1987. Serotonin stimulates DNA synthesis in fibroblasts acting through 5-HT1B receptors coupled to a Giprotein. Nature (Lond.). 335:254-256.

44. Ozaki, Y., M. P. Edelstein, and D. S. Duch. 1987. The actions of interferon and antiinflammatory agents on induction of indoleamine 2,3-dioxygenase in human peripheral blood monocytes. Biochem. Biophys. Res. Commun. 144:1147-1153. 